Z. Klin. Chem. Klin. Biochem.

13. Jg. 1975 , S. $163-167$

\title{
Untersuchungen zur vollständigen enzymatischen Hydrolyse von Steroidkonjugaten im Harn
}

\author{
Von V. Graef und M. Fuchs \\ Zentrum für Biochemie der Universität Gießen
}

(Eingegangen am 17. Januar/17. März 1975)

Bei der Inkubation von Harn mit $\beta$-Glucuronidase/Arylsulfatase aus Helix pomatia wird nur cine unvollständige Hydrolyse der Steroidkonjugate erreicht, da Harn Stoffe enthält, die diese Enzyme hemmen. Durch die Chromatographie des Harnes an einer Amberlite XAD-2-Säule ist eine Trennung der Hemmstoffe von den Steroidkonjugaten möglich. Mit dieser Methode läßt sich die vollständige enzymatische Hydrolyse der Steroidkonjugate im Harn in $24 \mathrm{~h}$ bei $37^{\circ} \mathrm{C}$ oder in $2 \mathrm{~h}$ bei $55^{\circ} \mathrm{C}$ durchführen.

\section{Studies on the complete enzymatic hydrolysis of steroid conjugates in urine}

The incubation of urine with $\beta$-glucuronidase/arylsulphatase from Helix pomatia results in the incomplete hydrolysis of urinary steroid conjugates, because inhibitors of these enzymes are present in urine. The inhibitors can be separated from the steroid conjugates by chromatography of the urine on a column of Amberlite XAD-2. By this method a complete enzymatic hydrolysis of urinary steroid conjugates is possible in 24 hours at $37^{\circ} \mathrm{C}$ or in 2 hours at $55^{\circ} \mathrm{C}$.

Die Metaboliten der Steroidhormone liegen im Harn in Form von Glucuroniden und Sulfaten vor, die vor der eigentlichen Bestimmung gespalten werden müssen. Das gelingt am schonendsten durch enzymatische Hydrolyse mit $\beta$-Glucuronidase aus Rinderleber, aus Bakterien oder aus Helix pomatia. Die letztgenannte Präparation enthält außer $\beta$-Glucuronidase auch Sulfatasen, die auch Sulfate von $3 \beta$-Hydroxysteroiden (z. B. Dehydroepiandrosteron) und Östrogenen spalten.

Man nimmt im allgemeinen an, daß bei der Inkubation des Harnes mit $\beta$-Glucuronidase die Steroidglucuronide vollständig gespalten werden. Dabei wird häufig der Umstand übersehen, daß Harn zahlreiche Hemmstoffe für die $\beta$-Glucuronidase (1-4) und für die Sulfatase $(5,6)$ enthält, die eine vollständige Spaltung der Steroidkonjugate verhindern können. In der vorliegenden Arbeit haben wir die Bedingungen für eine vollständige Hydrolyse der Steroidkonjugate im Harn untersucht. Drei Methoden wurden miteinander verglichen:

1. Inkubation von Nativharn mit $\beta$-Glucuronidase/Arylsulfatase aus Helix pomatia,

2. Abtrennung der Steroidkonjugate durch Chromatographie des Harnes an einer Säule aus Amberlite XAD-2 nach Bradlow (7) und anschließende Hydrolyse mit $\beta$-Glucuronidase/Arylsulfatase und

3. Extraktion der Steroidkonjugate aus dem Harn mit A ther/Ä thanol nach Kellie \& Wade (8) und anschliesse:2de enzymatische Hydrolyse.

\section{Material und Methoden}

\section{Reagenzien}

$\beta$-Glucuronidase/Arylsulfatase $(5,2 \mathrm{U}=100000 \mathrm{Fishman}$ Einheiten $\beta$-Glucuronidase/ml und 2,6 $U=57000$ Roy-Einheiten Arylsulfatase/ml): Fa. BOEHRINGER, Mannheim; Chromosorb W/AW-DMCS, 80-100 mesh, für Gaschromatographic; Östriol, Androsteron, Ätiocholanolon, $5 \beta$-Androstan-3, 17-dion, Dehydrocpiandrosteron (DHA), Dehydroepiandrosteronsulfat-Natriumsalz, Pregnandiol, Pregnantriol: Fa. E. MERCK, Darmstadt; Silicon XE-60, pract. für Gaschromatographie; Silicon SE-30, pract., für Gaschromatographie; N, O-Bis-(trimethylsilyl)acetamid, reinst; Trimethylchlorsilan, rcinst; Amberlite XAD-2 (300-1000 $\mu \mathrm{m})$ p.a.: Fa. SERVA, Heidelberg. Diäthyläther p. a. (Fa. E. MERCK, Darmstadt) wurde über Natriumdraht destilliert und in braunen Flaschen aufbewahrt. Alle übrigen Chemikalien stammen von der Firma E. MERCK, Darmstadt.

\section{Geräte}

Gaschromatograph F 20 der Fa. PERKIN-ELMER mit Flammenionisationsdetektor, Glassäulc von $2 \mathrm{~m}$ Länge mit $3 \%$ XE-60 auf Chromosorb W/AW-DMCS, Stickstoffstrom $46 \mathrm{ml} / \mathrm{min}$, synthetische Luft $300 \mathrm{ml} / \mathrm{min}$, Wasscrstoff $30 \mathrm{ml} / \mathrm{min}$, Säulentemperatur $210-230^{\circ} \mathrm{C}$, Einspritzblock $255-290^{\circ} \mathrm{C}$. Gaschromatograph 1525-C der Firma VARIAN mit Flammenionisationsdetektor, Glassäule von $2 \mathrm{~m}$ Länge mit 3\% SE-30 auf Chromosorb W/AW-DMCS, Stickstoffstrom $40 \mathrm{ml} / \mathrm{min}$, synthetische Luft $300 \mathrm{ml} / \mathrm{min}$, Wasserstoff $30 \mathrm{ml} / \mathrm{min}$, Säulentemperatur $260-270^{\circ} \mathrm{C}$, Einspritzblock $300^{\circ} \mathrm{C}$. Dieses Gerät wurde zur Östriol-Bestimmung verwendet. KompensationsPolarplanimeter zur Bestimmung der Peakflächen: Fa. A. OTT, Kempten.

Normalmethode zur enzymatischen Hydrolyse von Steroidkonjugaten

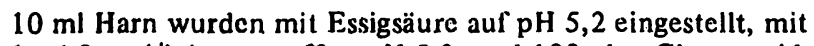
$1 \mathrm{ml} 2 \mathrm{~mol} / \mathrm{l}$ Acctatpuffer, pH 5,2, und $100 \mu \mathrm{l} \beta$-Glucuronidase/ 
Arylsulfatase versetzt und $24 \mathrm{~h}$ bei $37^{\circ} \mathrm{C}$ inkubiert. Der Harn wurde nach dem Erkalten einmal mit $40 \mathrm{ml}$ und einmal mit $20 \mathrm{ml}$ Äther ausgeschüttelt. Die vereinig ten Extrakte wurden einmal mit $10 \mathrm{ml} 1 \mathrm{~mol} / 1 \mathrm{NaOH}$ und zweimal mit je $10 \mathrm{ml}$ Wasser ausgeschüttelt und am Vakuum-Rotationsverdampfer bei $40^{\circ} \mathrm{C}$ eingedampft. Reste von Wasser wurden mit $5 \mathrm{ml}$ Äthanol im Vakuum abgedampft. Den Verdampfungsrückstand überfuhrten wir mit zweimal $2 \mathrm{ml}$ Methanol in ein spitzes Zentrifugenröhrchen und verdampften das Lösungsmittel im Stickstoffstrom bei $50^{\circ} \mathrm{C}$. In diesem Röhrchen wurden dann 100 bzw. $200 \mu 1$ 5 $\beta$-Androstan-3,17-dion-Lösung ( $1 \mathrm{~g} / 1$ Äthanol) verdampft. Dann ließen wir $1 \mathrm{ml}$ Äthanol/Benzol (Volumina, $2 \mathrm{ml}+7 \mathrm{ml}$ ) an der Innenwand des Röhrchens herunterlaufen, um die Steroide in die Spitze zu bringen. Das Lösungsmittel wurde ebenfalls mit trockenem Stickstoff bei $50^{\circ} \mathrm{C}$ abgeblasen und der Rückstand in $50 \mu \mathrm{l}$ Schwefelkohlenstoff aufgenommen. $1 \mu$ der Lösung wurde in den Gaschromatographen eingespritzt.

Extraktion der Steroidkonjugate aus dem Harn nach Kellie und Wade (8)

$10 \mathrm{ml}$ Harn, in dem $5 \mathrm{~g} \mathrm{Ammoniumsulfat} \mathrm{gelöst} \mathrm{waren,} \mathrm{wurden}$ dreimal mit je $10 \mathrm{ml}$ Âther/Äthanol (Volumina, $30 \mathrm{ml}+10 \mathrm{ml}$ ) ausgeschüttelt. Zur Trennung der Phasen war eine Zentrifugation bei $4500 \mathrm{U} / \mathrm{min}$ nötig. Die vereinigten organischen Extrakte wurden durch ein mit Äther/Äthanol angefeuch tetes Faltenfilter in einen $100 \mathrm{ml}$-Rundkolben filtriert. Das Filter wurde mit Äther/Äthanol nachgespült. Nach Verdampfen des Lösungsmittels am Vakuum-Rotationsverdampfer bei $40^{\circ} \mathrm{C}$ wurde der Rückstand in $10 \mathrm{ml}$ dest. Wasser und $1 \mathrm{ml} 2 \mathrm{~mol} / 1$ Acetatpuffer, pH 5,2, gelöst und mit $100 \mu \mathrm{l} \beta$-Glucuronidase/Arylsulfatase $24 \mathrm{~h}$ bei $37^{\circ} \mathrm{C}$ inkubiert. Die weitere Aufarbeitung erfolgte wie bei der Normalmethode.

Am berlite-Säulen methode zur Extraktion von Steroidkonjugaten aus dem $\mathrm{Harn}$

100 g Amberlite XAD-2 wurden zur Entfernung niedermolekularer Bestandteile dreimal mit je $500 \mathrm{ml}$ Methanol p. a. 2 min geschüttelt. Das überstehende Methanol wurde nach 5 min abgegossen. Anschließend wurde das Amberlite-Harz dreimal mit je $500 \mathrm{ml}$ dest. Wasser je 2 min durchgeschüttelt und in wäßriger Suspension aufbewahrt. Benutztes Amberlite kann durch Schütteln mit Methanol und Wasser in der gleichen Weise regeneriert werden. In eine Glassäule $(48 \times 1 \mathrm{~cm}) \mathrm{mit}$ Hahn, die unten mit feuchter Watte verschlossen war, wurde soviel Amberlite gefüllt, daß die Füllhöhe $8 \mathrm{~cm}$ betrug.

Auf die Amberlite-Säule wurden $10 \mathrm{ml}$ Harn pipettiert. Nach dem Einsickern des Harnes wurde mit $10 \mathrm{ml}$ dest. Wasser nachgewaschen, Harn und Waschwasser wurden verworfen. Die an das Amberlite-Harz adsorbierten Steroide, Steroidglucuronide und Steroidsulfate wurden mit $20 \mathrm{ml}$ Methanol eluiert. Das Eluat dampften wir im Rundkolben am Rotationsverdampfer bei $40^{\circ} \mathrm{C}$ im Vakuum zur Trockne ein. Der Rückstand wurde in $10 \mathrm{ml}$ dest. Wasser und $1 \mathrm{ml} 2 \mathrm{~mol} / \mathrm{l}$ Acetatpuffer, pH 5,2, gelöst und mit $100 \mu \mathrm{l} \beta$-Glucuronidase/Arylsulfatase $24 \mathrm{~h}$ bei $37^{\circ} \mathrm{C}$ inkubiert. Die weitere Aufarbeitung erfolgte wie bei der Normalmethode.

\section{Östriol-Bestimmung im Harn schwangerer Frauen}

$10 \mathrm{ml}$ Harn wurden, wie oben beschrieben, an einer AmberliteSäule gereinigt. Die Waschwassermenge betrug hierbei nur $5 \mathrm{ml}$. Die im Methanol-Eluat enthaltenen Steroidkonjugate wurden nach Verdampfen des Methanols in $10 \mathrm{ml}$ Wasser und $1 \mathrm{ml}$

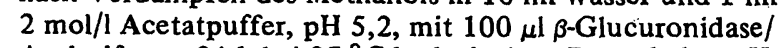
Arylsulfatase $24 \mathrm{~h}$ bei $37^{\circ} \mathrm{C}$ hydrolysiert. Das erkaltete Hydrolysat wurde mit $2,5 \mathrm{ml} 10 \mathrm{~mol} / 1 \mathrm{NaOH}$ versetzt und in einen Schütteltrichter überführt. Der Kolben wurde mit $3 \mathrm{ml}$ dest. Wasser nachgespült. Die alkalische Lösung wurde zur Entfernung neutraler Steroide mit $30 \mathrm{ml}$ Äther ausgeschüttelt und in einen zweiten Schütteltrichter überführt. Der Äther wurde mit $3 \mathrm{ml}$ $1 \mathrm{~mol} / \mathrm{l} \mathrm{NaOH}$ nachextrahiert und dann verworfen. Die vereinigten alkalischen Lösungen säuerten wir mit $3 \mathrm{ml}$ konz. Sạlzsäure an und extrahierten sie zweimal mit je $30 \mathrm{ml}$ eiskaltem $\ddot{A}$ ther. Die vereinigten Äther-Extrakte wurden einmal mit $5 \mathrm{ml}$ Carbonatpuffer, pH 10,0, und zweimal mit je $5 \mathrm{ml}$ dest. Wasser ausgeschüttelt und eingedampft. Reste von Wasser wurden durch Abdampfen mit $5 \mathrm{ml}$ Athanol im Vakuum entfernt. Den Verdampfungsrückstand überführte man mit zweimal $2 \mathrm{ml}$ Methanol in ein Spitzröhrchen, in dem die Lösung im Stickstoffstrom bei $50^{\circ} \mathrm{C}$ zur Trockne gedampft wurde. Der Rückstand wurde mit $0,2 \mathrm{ml}$ Pyridin und $0,5 \mathrm{ml}$ Acetanhydrid $1 \mathrm{~h}$ auf $65^{\circ} \mathrm{C}$ erwärmt, worauf das Acetylierungsgemisch im Stickstoffstrom bei $50^{\circ} \mathrm{C}$ abgeblasen worde. Nach Zusatz von $200 \mu \mathrm{l}$ internem Standard ( $1 \mathrm{~g} / 1$ Cholesterinace tat in Aceton) wurde erneut mit Stickstoff eingedampft. Wir nahmen den Rückstand in $100 \mu l$ Aceton auf und spritzten $1 \mu l$ dieser Lösung in den Gaschromatographen ein.

Dünnschichtchromatographische Vorreinigung des Harnextraktes

Zur Abtrennung von Androsteron, Ätiocholanolon und Dehydroepiandrosteron wurde der nach Amberlite-Extraktion und enzymatischer Hydrolyse gewonnene Harnextrakt mit zweimal $100 \mu$ l Aceton punktförmig $1,5 \mathrm{~cm}$ vom unteren Rand einer Kicselgel G-Dünnschichtplatte aufgetragen. Daneben trugen wir je 10-15 $\mu \mathrm{g}$ der reinen Steroide auf. Nach Entwickeln der Platte im Laufmittel Chloroform/Äthanol (Volumina $95 \mathrm{ml}+$ $5 \mathrm{ml}$ ) wurden die Flecken der Referenzsubstanzen mit einer Mischung aus 2 Vol $20 \mathrm{~g} / 1$ alkohol. $m$-Dinitrobenzol-Lösung und $1 \mathrm{Vol}$ methanol. $\mathrm{KOH}(7,5 \mathrm{~g}$ in $50 \mathrm{ml}$ Methanol) angesprüht und mit einem Föhn erwärmt. Androsteron, Ätiocholanolon und Dehydroepiandrosteron des Harnextraktes wurden zusammen ausgekratzt, das Kieselgel wurde dreimal mit je $2 \mathrm{ml}$ Methanol eluiert.

Zur Isolierung von Pregnandiol aus dem Harnextrakt verwendeten wir Kieselgel G-Platten, die im Laufmittel Chloroform/ Äthanol (Volumina $90 \mathrm{ml}+10 \mathrm{ml}$ ) entwickelt wurden. Der Vergleichsfleck des neben dem Harnextrakt aufgetragenen reinen Pregnandiols wurde sichtbar gemacht, indem die Platte kurz in eine Kammer mit Joddampf gestellt wurde. Pregnantriol wurde aus dem Harnextrakt auf Kieselgel G-Dünnschichtplatten abgetrennt, die in Chloroform/Aceton (Volumina $50 \mathrm{ml}$ $+50 \mathrm{ml}$ ) entwickelt wurden. Die Steroide wurden mit dreimal $2 \mathrm{ml}$ Methanol aus dem ausgekratzten Kieselgel eluiert.

\section{Ergebnisse}

Überprüfung der Adsorptionsfähigkeit des Amberlite-Harzes für Steroidkonjugate

Um das Adsorptionsvermögen des Amberlite XAD=2 für Steroidsulfate zu prüfen, gaben wir je $8 \mathrm{mg}$ Östronsulfat bzw. Dehydroepiandrosteronsulfat-Natrium in je $16 \mathrm{ml}$ Wasser auf die Amberlite-Säule, die anschliessend mit je $10 \mathrm{ml}$ Wasser nachgewaschen wurde. In den wäßrigen Eluaten fanden wir nạch Inkubation mit $\beta$-Glucuronidase/Arylsulfatase keine Steroide. Die eingesetzten Steroidsulfate ließen sich vollständig mit $20 \mathrm{ml}$ Methanol von der Säule eluieren.

Um zu prüfen, ob auch Steroidglucuronide fest an das Amberlite-Harz adsorbiert werden und ob sie sich durch Wasser eluieren lassen, gaben wir $10 \mathrm{ml} \mathrm{Harn} \mathrm{auf} \mathrm{eine}$ Amberlite-Säule, die mit $10 \mathrm{ml}$ Wasser nachgewáschen wurde. Harn und Waschwasser wurden mit Ammoniumsulfat gesättigt und mit $\ddot{A}$ ther/Äthanol extrahiert. In diesem Extrakt konnten wir nach enzymatischer Hydrolyse keine Steroide nachweisen. Durch Elution der Amberlite-Säule mit $20 \mathrm{ml}$ Methanol wurden alle Steroidkonjugate heruntergelöst.

Während die Konjugate von Androsteron, Ä tiocholanolon, Dehydroepiandrosteron, Pregnandiol und Pregnan- 
triol fest an Amberlite gebunden wurden und sich durch Waschen mit Wasser nicht eluieren ließen, wurden die Konjugate des Östriols weniger fest an das Amberlite gebunden. Wir analysierten 2 Harne von schwangeren Frauen, die 310 bzw. $207 \mu \mathrm{g}$ Östriol/10 ml enthielten, mit der Amberlite-Methode. Durch Nachwaschen der Säule mit $5 \mathrm{ml}$ Wasser wurden 1,6\% und durch Waschen mit $20 \mathrm{ml}$ Wasser 7,6\% konjugiertes Östriol von der Säule eluiert.

Vergleich der Normalmethode mit der $\ddot{A}$ ther/ $\ddot{A}$ thanol-Extraktionsmethode und der A mberlite-Methode

Wir haben 10 Harne nach der Normalmethode (Inkubation von Nativharn mit $\beta$-Glucuronidase/Arylsulfatase), nach der Amberlite-Methode und nach der Äther/ Äthanol-Extraktionsmethode analysiert. Bei den beiden letztgenannten Methoden wurden die Hemmstoffe für $\beta$-Glucuronidase und Sulfatase abgetrennt, was zu einer besseren Spaltung der Steroidkonjugate führte (Tab. 1). Die höchsten Steroidkonzentrationen ermittelten wir mit der Amberlite-Methode. Die nach der Ä ther/Ä thanolExtraktionsmethode gefundenen Steroidkonzentrationen lagen in allen Fällen unter den mit der AmberliteMethode gefundenen Werten. Aus den Angaben in
Tab. 1. Vergleich der Stcroidbestimmungen in 10 Harnen mit der Normalmethode, der $\ddot{A}$ ther/Äthanol-Ex traktionsmethode und der Amberlite-Methode ( $=100 \%$ ).

\begin{tabular}{|c|c|c|}
\hline \multirow[t]{2}{*}{ Steroid } & \multicolumn{2}{|c|}{ Steroide gefunden im Vergleich zur } \\
\hline & $\begin{array}{l}\text { Normal- } \\
\text { methode }(\%)\end{array}$ & $\begin{array}{l}\text { Äther/Äthanol- } \\
\text { Methode (\%) }\end{array}$ \\
\hline Androsteron & $71,2 \pm 14,2$ & $86,9 \pm 6,0$ \\
\hline Ätiocholanolon & $74,0 \pm 17,3$ & $87,3 \pm 10,9$ \\
\hline Dehydroepiandrosteron & $21,2 \pm 7,5$ & $61,3 \pm 23,6$ \\
\hline Pregnandiol & $63,8 \pm 18,0$ & $88,2 \pm 9,9$ \\
\hline Pregnantriol & $72,0 \pm 17,0$ & $82,8 \pm 13,1$ \\
\hline
\end{tabular}

Tabelle 1 ist zu ersehen, daß die Spaltung der Steroidkonjugate nach der Normalmethode unvollständig ist. Besonders schlecht wurde Dehydroepiandrosteron-Sulfat gespalten, da im Harn enthaltene Phosphat- und Sulfationen die Sulfatase stark hemmen.

Um zu prüfen, ob mit der dreifachen Enzymmenge (300 $\mu \mathrm{l} \beta$-Glucuronidase/Arylsulfatase/10 ml Harn) eine vollständige Hydrolyse der Steroidkonjugate in Nativharn möglich ist, haben wir 5 Harne mit 100 bzw $300 \mu$ l $\beta$-Glucuronidase/Arylsulfatase nach der Normalmethode und nach der Amberlite-Methode (mit $100 \mu \mathrm{l}$ Enzym) analysiert. Tabelle 2 zeigt, daß sich die enzymatische Hydrolyse in vielen Fällen zwar verbessern läßt,

Tab. 2. Vergleich der Normalmethode (unterschiedliche Mengen $\beta$-Glucuronidase/Arylsulfatase) mit der Amberlite-Methode.

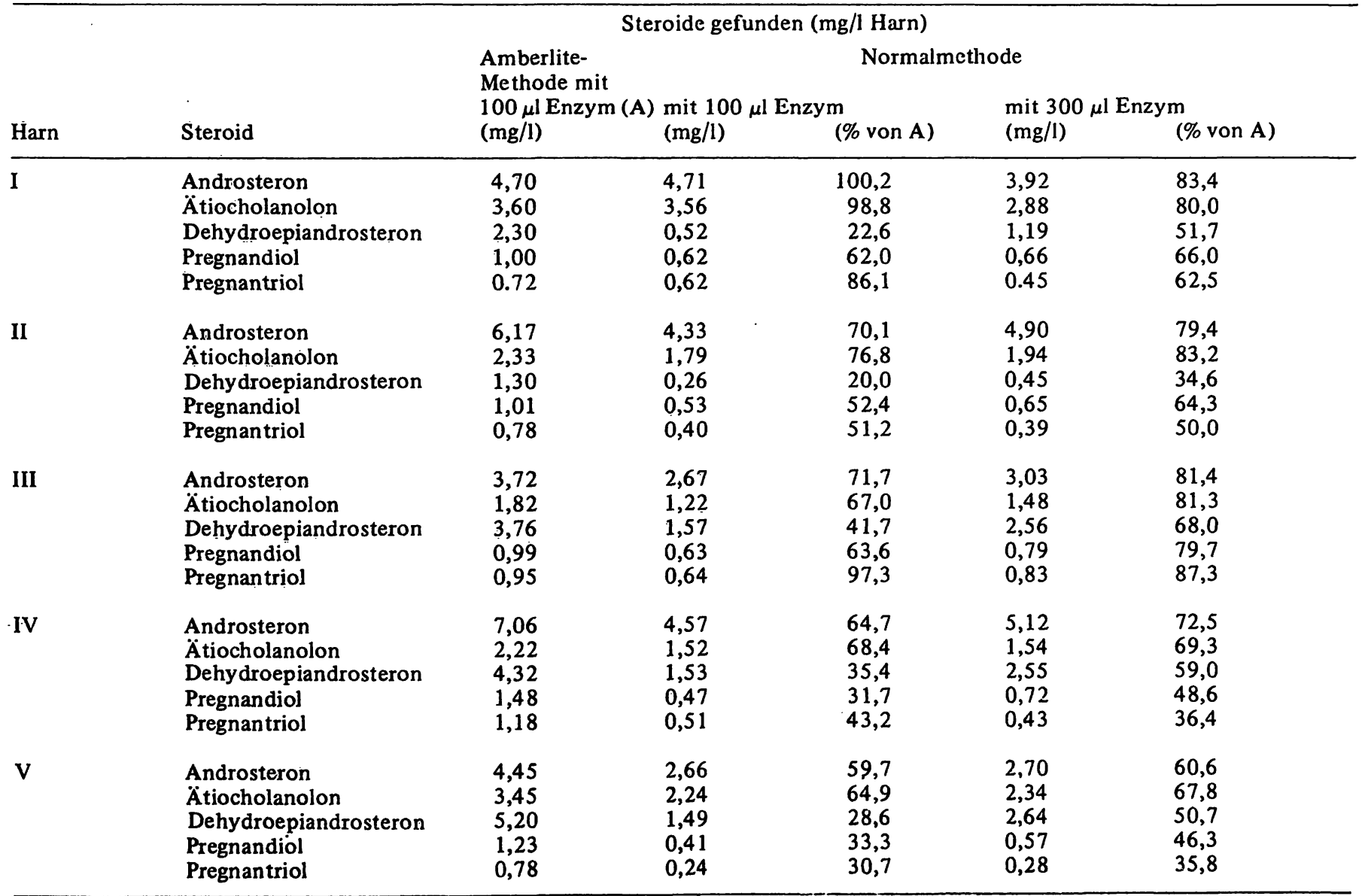


wenn die Harne mit der dreifachen Enzymmenge inkubiert wurden, doch lagen die Werte immer noch deutlich unter den mit der Amberlite-Methode gewonnenen Werten. In einigen Fällen führte eine Vergrößerung der Enzymmenge bei der Normalmethode auch zu einer Verschlechterung der Hydrolyse. Das wurde bereits von Kornel (9) bei der Hydrolyse von Corticosteroid-glucuroniden mit $\beta$-Glucuronidase aus Rinderleber beobachtet.

\section{Inkubationsdauer}

Da durch die Vorreinigung des Harnes an Amberlite die Hemmstoffe der $\beta$-Glucuronidase und der Sulfatase entfernt wurden, erschien es möglich, die Steroidkonjugate auch in kürzeren Zeiten zu spalten. Zwei Harne wurden mit der Amberlite-Methode 6, 12, 18 und $24 \mathrm{~h}$ bei $37^{\circ} \mathrm{C}$ mit je $100 \mu$ l $\beta$-Glucuronidase/Arylsulfatase inkubiert. Wir fanden, daß zur vollständigen Hydrolyse von Androsteron-, Ä tiocholanolon- und Pregnandiolglucuronid und von Dehydroepiandrosteron-Sulfat eine Inkubationszeit von $24 \mathrm{~h}$ nötig ist.

Um die Hydrolysedauer der nach Abtrennung der Hemmstoffe aus Harn mit Amberlite extrahierten Steroidkonjugate zu verkǜzzen, haben wir die enzymatische Hydrolyse bei erhöhter Temperatur durchgeführt. Wir fanden, daß das Temperaturoptimum für die $\beta$-Glucuronidase (mit Phenolphthalein-glucuronid als Substrat) bei $55^{\circ} \mathrm{C}$ und für die Arylsulfatase (mit Phenolphthaleindisulfat als Substrat) bei $45^{\circ} \mathrm{C}$ liegt. Da aber Steroidglucuronide und -sulfate gleichzeitig gespalten werden sollten, war zu prüfen, ob Dehydroepiandrosteronsulfat sich auch bei $55^{\circ} \mathrm{C}$ in $2 \mathrm{~h}$ vollständig spalten läßt. Wir fanden, daß 25-150 $\mu$ g Dehydroepiandrosteronsulfat mit $100 \mu \mathrm{l} \beta$-Glucuronidase/Arylsulfatase in $2 \mathrm{~h}$ bei $55^{\circ} \mathrm{C}$ zu durchschnittlich $98,5 \%$ hydrolysiert wurden.

Eine Schnellhydrolyse ist besonders für die Östriol-Bestimmung im Harn schwangerer Frauen erforderlich. Wir haben 9 Harne (je $10 \mathrm{ml}$ ) nach Entfernung der Hemmstoffe durch Amberlite-Chromatographie mit $200 \mu \mathrm{l} \beta$-Glucuronidase/Arylsulfatase 2 und $6 \mathrm{~h}$ bei $55^{\circ} \mathrm{C}$ und $24 \mathrm{~h}$ bei $37^{\circ} \mathrm{C}$ inkubiert. Verglichen mit

Tab. 3. Östriol-Bestimmung im Harn schwangerer Frauen nach der Amberlite-Methode. Vergleich der enzymatischen Hydrolyse bei $55^{\circ} \mathrm{C}$ und bei $37^{\circ} \mathrm{C}$.

Östriol (mg/l Harn) gefunden nach einer Inkubationszeit von

\begin{tabular}{llll} 
Harn & $2 \mathrm{~h}\left(55^{\circ} \mathrm{C}\right)$ & $6 \mathrm{~h}\left(55^{\circ} \mathrm{C}\right)$ & $24 \mathrm{~h}\left(37^{\circ} \mathrm{C}\right)$ \\
\hline I & 13,4 & 13,1 & 12,8 \\
II & 22,1 & 22,6 & 22,9 \\
III & 23,7 & 22,9 & 22,5 \\
IV & 13,3 & 13,8 & 13,6 \\
V & 22,0 & 22,1 & 22,6 \\
VI & 10,0 & 9,6 & 9,6 \\
VII & 33,5 & - & 33,8 \\
VIII & 34,8 & - & $35,6$. \\
IX & 24,6 & 24,6 & 25,0 \\
\hline
\end{tabular}

der $24 \mathrm{~h}$ Hydrolyse bei $37^{\circ} \mathrm{C}$ fanden wir nach $2 \mathrm{~h}$ bei $55^{\circ} \mathrm{C} 100,1 \pm 3,5 \%$ und nach $6 \mathrm{~h}$ bei $55^{\circ} \mathrm{C} 100,0 \pm$ $1,8 \%$ Östriol (Tab. 3).

Präzision und Richtigkeit der Amberlite-Methode Zur Prüfung der Präzision der Methode wurden je $10 \mathrm{ml}$ desselben $24 \mathrm{~h}$-Harnes einer männlichen Versuchsperson zwölfmal analysiert. Zur Prüfung der Präzision der Östriol-Bestimmung wurden je $10 \mathrm{ml}$ Harn einer schwangeren Frau sechsmal analysiert. Die Standardabweichungen und Variationskoeffizienten für alle Steroide sind in Tabelle 4 zusammengefaßt.

Tab. 4. Präzision der gaschromatographischen Steroidbestimmung im Harn nach der Amberlite-Me thode

\begin{tabular}{lrrrl}
\hline Steroid & $\mathrm{n}$ & $\overline{\mathbf{x}}(\mathrm{mg} / \mathrm{l})$ & $\mathrm{s}^{\prime}(\mathrm{mg} / \mathrm{l})$ & $\mathrm{VK}(\%)$ \\
\hline Androsteron & 12 & 3,10 & 0,160 & 5,2 \\
Ätiocholanolon & 12 & 1,66 & 0,106 & 6,4 \\
Dehydroepiandrosteron & 12 & 5,72 & 0,280 & 4,9 \\
Pregnandiol & 12 & 1,22 & 0,096 & 7,9 \\
Pregnantriol & 12 & 0,93 & 0,085 & 9,1 \\
Östriol & 6 & 12,90 & 0,527 & 4,0 \\
\hline
\end{tabular}

In der Regel enthalten die Gaschromatogramme der Harnextrakte keine weiteren Peaks von Substanzen, die ähnliche Retentionszeiten besitzen wie Androsteron, Ätiocholanolon, Dehydroepiandrosteron, Pregnandiol und Pregnantriol, so daß sich eine weitere Vorreinigung der Harnextrakte erübrigt. In einzelnen Fällen enthielten die Gaschromatogramme aber noch zusätzliche Peaks in der Nähe der Peaks der genannten Steroide, so daß es z. B. zur Schulterbildung kam. In diesen Fällen haben wir die Harnextrakte durch Dünnschichtchromatographie vorgereinigt.

Wir haben die Wiederfindungsraten für die einzelnen Schritte untersucht. Die Wiederfindung nach AmberliteSäulenchromatographie betrug für Androsteron 99,6\%, für Pregnantriol 99,0\% und für Tetrahydrocortisol $99,7 \%$. Der Verlust dürfte auch bei anderen $\mathrm{C}_{19}$ - und $\mathrm{C}_{21}$-Steroiden nicht höher als $1 \%$ sein. Beim Östriolglucuronid beträgt er $1,6 \%$, wenn die Amberlite-Säule

Tab. 5. Bestimmung der Wiederfindungsrate nach Äther-Extraktion und nach Dünnschichtchromatographie

\begin{tabular}{lllc}
\hline & $\begin{array}{l}\text { Wiederfindung } \\
\text { nach Extraktion } \\
\text { Steroid }\end{array}$ & $\mathrm{n}(\%)$ & $\begin{array}{l}\text { Wiederfindung nach } \\
\text { Dünnschichtchro- } \\
\text { matographie (\%) }\end{array}$ \\
\hline $\begin{array}{lll}\text { Androsteron } \\
\text { Ätiocholanolon }\end{array}$ & 6 & $98,4 \pm 2,4$ & $97,1 \pm 1,9$ \\
$\begin{array}{l}\text { Dehydroepi- } \\
\text { androsteron }\end{array}$ & 6 & $94,7 \pm 1,3$ & $95,7 \pm 2,2$ \\
Pregnandiol & 6 & $98,9 \pm 1,7$ & $93,4 \pm 2,0$ \\
Pregnantriol & 6 & $97,2 \pm 4,8$ & $100,9 \pm 1,0$ \\
\hline
\end{tabular}


mit $5 \mathrm{ml}$ Wasser gewaschen wird. Die Wiederfindung nach Extraktion der Steroide mit Ä ther (je $50 \mu \mathrm{g}$ Steroid/10 ml Wasser) und U'berführen des Extraktes in Spitzröhrchen wurde zusammen bestimmt (Tab. 5). Die Wiederfindung für die gesamte Östriol-Bestimmungsmethode mit Vorreinigung des Harnes an einer Amberlite-Säule betrug $85,0 \%$.

\section{Diskussion}

Die im Harn enthaltenen Steroidkonjugate lassen sich zwar durch $\beta$-Glucuronidase und Sulfatase am schonendsten hydrolysieren, doch ist damit zu rechnen, daß infolge der Anwesenheit von Hemmstoffen für diese Enzyme im Harn die Hydrolyse nicht vollständig ist. Um das Ausmaß dieser Hemmung zu untersuchen, haben wir die enzymatische Hydrolyse nach drei verschiedenen Methoden durchgeführt.

1. Nativharn wurde mit $\beta$-Glucuronidase/Arylsulfatase inkubiert (Normalmethode).

2. Die gleichen Harne wurden nach einer erstmals von Bradlow (7) beschriebenen Methode mit Amberlite XAD-2, einem neutralen Polystyrolharz, behandelt. Dabei wurden die Steroidkonjugate an Amberlite adsorbiert und konnten so von den Hemmstoffen getrennt werden. Die Steroidkonjugate wurden mit Methanol vom Amberlite eluiert nnd anschließend enzymatisch mit $\beta$-Glucuronidase/Arylsulfatase hydrolysiert.

3. Die Steroidkonjugate wurden aus dem mit Ammoniumsulfat gesättigten Harn mit $\ddot{A}$ ther/ $\ddot{A}$ thanol nach
Kellie \& Wade (8) extrahiert und auf diese Weise von den gut wasserlöslichen Hemmstoffen getrennt.

Die Steroidkonjugate wurden dann enzymatisch gespalten.

Beim Vergleich der 3 Methoden wurde deutlich, daß bei der Inkubation von Nativharn mit $\beta$-Glucuronidase/ Arylsulfatase nur eine unvollständige Spaltung der Steroidkonjugate erreicht wurde. Auch mit der dreifachen Enzymmenge wurde keine vollständige Hydrolyse erreicht. Außerdem ist die Hydrolyse mit zu großen Enzymmengen oder auch eine Verlängerung der Hydrolysedauer nicht zu empfehlen, da die meisten Präparationen der $\beta$-Glucuronidase noch Fremdaktivitäten enthalten, die Steroide chemisch verändern können $(2,10,11)$. Durch die Amberlite-Methode wurde eine vollständige Trennung der Steroidkonjugate von den Hemmstoffen erreicht. Die Steroidkonjugate ließen sich mit $\beta$-Glucuronidase/Arylsulfatase vollständig spalten. Diese Methode läßt sich am schnellsten und einfachsten durchführen und liefert die höchsten Ausbeuten an Steroiden. Zur vollständigen Hydrolyse bei $37^{\circ} \mathrm{C}$ ist eine Inkubationsdauer von $24 \mathrm{~h}$ erforderlich. Durch Erhöhung der Inkubationstemperatur auf $55^{\circ} \mathrm{C}$ lassen sich die Steroidkonjugate bereits in $2 \mathrm{~h}$ vollständig spalten. Diese Schnellhydrolyse ist von besonderer Bedeutung für die ÖstriolBestimmung im Harn schwangerer Frauen. Die in der verwendeten Enzympräparation aus Helix pomatia enthaltene Sulfatase spaltet nur die Sulfate von $3 \beta$-Hydroxysteroiden und Östrogenen, nicht jedoch $3 \alpha$-Hydroxysteroidsulfate. Um auch diese meist nur in geringer Menge im Harn enthaltenen Sulfate zu spalten, muß sich der enzymatischen Hydrolyse noch die Solvolyse anschließen.

\section{Literatur}

1. Karunairatnam, M. C. \& Levvy, G. A. (1949), Biochem. J. 44, 599-604.

2. Slaunwhite Jr., W. R. \& Sandberg, A. V. (1960), Endocrinology 67, 815-828.

3. Dohrmann, R. E. (1969), $\beta$-Glucuronidase, Springer-Verlag Berlin-Heidelberg-New York.

4. Sakamoto, W. \& Nisihikaze, O. (1972), Enzyme 13, 211 223.

5. Roy, A. B. (1956), Biochem. J. 62, 41-49.
6. Roy, A. B. (1957), Biochem. J. 66, 700-703.

7. Bradlow, H. L. (1968), Steroids 11, 265-272.

8. Kellie, A. E. \& Wade, A. P. (1957), Biochem. J. 66, 196206.

9. Kornel, L. (1960), Metabolism 9, 82-84.

10. Vestergaard, P. (1962), Acta Endocrinol. (Copenhagen) Suppl. 64, 50-68.

11. Roy, S. \& Slaunwhite Jr., W. R. (1970), Steroids 16, 69-78.

\author{
Dr. V. Graef \\ Zentrum f. Biochemie \\ am Klinikum d. \\ Justus Liebig-Univ. \\ 63 Gießen \\ Friedrichstr. 24
}


